\title{
Gradhiva
}

GRADHI

Revue d'anthropologie et d'histoire des arts

1 | 2005

Haïti et l'anthropologie

\section{Anthropologie, politique et engagement social}

L'expérience du Bureau d'ethnologie d'Haïti

Political anthropology and social involvement

Rachelle Charlier-Doucet

\section{CpenEdition}

Journals

Édition électronique

URL : http://journals.openedition.org/gradhiva/313

DOI : 10.4000/gradhiva.313

ISSN : 1760-849X

Éditeur

Musée du quai Branly Jacques Chirac

Édition imprimée

Date de publication : 1 mai 2005

Pagination : 109-125

ISBN : 2-915133-08-5

ISSN : 0764-8928

Référence électronique

Rachelle Charlier-Doucet, « Anthropologie, politique et engagement social », Gradhiva [En ligne],

1 | 2005, mis en ligne le 10 décembre 2008, consulté le 05 mai 2019. URL : http://

journals.openedition.org/gradhiva/313; DOI : 10.4000/gradhiva.313

Ce document a été généré automatiquement le 5 mai 2019.

(c) musée du quai Branly 


\title{
Anthropologie, politique et engagement social
}

\author{
L'expérience du Bureau d'ethnologie d'Haïti \\ Political anthropology and social involvement
}

Rachelle Charlier-Doucet

À Lucien Smarth, ethnologue haïtien.

Pour que se concrétise le rêve de renaissance du Bureau d'ethnologie.

Je tiens à remercier ici tous ceux qui ont bien voulu partager avec moi leur expérience du Bureau et leur connaissance de l'ethnologie et de l'histoire haïtienne: Jean-Yves Blot, Michel Hector, Michel Lamartinière Honorat, Lorraine Mangonès, entre autres. Mes remerciements vont également à $M$. Tardieu, conservateur de la Bibliothèque haïtienne des pères du Saint-Esprit (St Martial), qui a mis à ma disposition les archives du fonds haitien, et à tous les membres du Bureau national d'ethnologie pour leur accueil, en particulier M. Arnold Dormilus et Mme Phanord, responsables de la bibliothèque.

1 Parler du Bureau d'ethnologie d'Haïti ${ }^{1}$ aujourd'hui est une tâche à la fois délicate et nécessaire. Nécessaire, car il faut tirer de l'oubli une institution qui a marqué le paysage intellectuel, social et politique d'Haïti au $\mathrm{xx}^{\mathrm{e}}$ siècle. Le Bureau d'ethnologie, souvent confondu avec la Faculté d'ethnologie, a très mauvaise presse aujourd'hui dans le pays. Il est évoqué, de manière confuse chez les uns, plus précise chez les autres, à la fois comme le bastion du duvaliérisme et le foyer de la religion vodou, laquelle est souvent décriée parce qu'associée à des pratiques de sorcellerie. La tâche s'avère donc délicate, car pour parler du Bureau et faire avec impartialité le bilan de ses soixante-deux années d'existence, il faut évoquer des moments douloureux de l'histoire nationale et toucher à des domaines encore sensibles dans la société haïtienne d'aujourd'hui. Le propos ici n'est pas de faire une analyse en profondeur de la production ethnographique de cette institution - d'autres contributions dans ce volume le feront - mais plutôt de retracer les grandes lignes de son histoire intellectuelle et tenter d'expliquer les causes du 
rayonnement puis du déclin de cette institution qui se voulait le «bastion de la connaissance scientifique haïtienne ».

\section{Contexte idéologique, politique et social de la création du Bureau}

2 Pour comprendre l'histoire du Bureau d'ethnologie et son poids idéologique dans le panorama intellectuel haïtien, il faut remonter à une trentaine d'années avant sa création, plus précisément à l'année $1915^{2}$. Le pays, déchiré par des luttes intestines incessantes est alors occupé militairement par les États-Unis. Cet événement, qui a infléchi l'histoire du pays, provoque un sursaut et une prise de conscience dans certains secteurs de la société. Car il fallait chercher les causes de la "gifle " pour mieux comprendre et réparer l'outrage. Très vite, des membres des classes paysannes prennent les armes : les plus farouches sont les "cacos », menés par Charlemagne Péralte. Préférant la plume aux armes, certains membres de la bourgeoisie noire et mulâtre organisent la résistance sur les fronts politique et culturel. Jean Price-Mars, avec la publication de Ainsi parla l'Oncle (1928), s'affirme comme l'une des figures de proue d'un nouveau « nationalisme culturel » fondé sur la valorisation de l'héritage africain. D'autres figures s'imposent également, notamment Jacques Roumain, de la Revue Indigène (1927), et les jeunes de la revue Les Griots (1938-1939) ayant à sa tête les «trois D»: Louis Diaquoi, Lorimer Denis et François Duvalier ${ }^{4}$. Entre tous ces groupes, que l'on peut situer comme appartenant à la "mouvance indigéniste » (Trouillot 1993), il y a certes une position commune autour de ce «nationalisme culturel » vaguement défini, mais il existe surtout de pofondes divergences idéologiques quant à la lecture de l'histoire et de l'organisation sociale haïtienne, divergences qui tiennent principalement à leurs origines sociales différentes ${ }^{5}$. Dans la foulée de l' " indigénisme ", proposition esthétique ${ }^{6}$, émergeront des doctrines sociales et politiques dont il faudrait se garder d'établir trop hâtivement des filiations conceptuelles. La question de couleur, qui a toujours été une pomme de discorde dans la société haïtienne, est à nouveau posée dans toute son acuité par Les Griots. Alors que la Revue Indigène disparaît, le groupe des Griots se renforce. Il articule une nouvelle vision de l'identité haïtienne, fondée sur une définition de la race, conçue non plus seulement comme une catégorie biologique, mais surtout et avant tout, comme une catégorie sociale et culturelle ${ }^{7}$. Des débats sur l'histoire, la culture, la nation ont lieu entre les membres des clubs sociaux, littéraires et politiques qui fleurissent; des polémiques s'engagent, qui s'intensifieront pendant les années 1940. C'est sur ce fond d'agitation politique, de revendications sociales et de quête identitaire que le 31 octobre 1941 est fondé le Bureau d'ethnologie de la République d'Haïti.

3 La cause première de cette création est, sans nul doute, la répression dont sont victimes les pratiquants de la religion vodou. Pendant les années 1941-1942, l'Église catholique romaine, appuyée par le gouvernement du président Élie Lescot, lança une croisade antivodou, officiellement appelée "campagne anti-superstitieuse " dont une première opération, de moindre envergure, avait déjà été déclenchée au XIX siècle. Le clergé catholique, alors composé d'étrangers (particulièrement de religieux bretons), entreprit de détruire systématiquement tous les objets cultuels des oumfo (temples vodou). Les pratiquants furent pourchassés et forcés de prendre une "carte» indiquant qu'ils rejetaient leur foi, d'où le terme créole « rejete » couramment employé par la population pour désigner cette campagne. Ces abus révoltèrent nombre de nationalistes haïtiens, et 
certains passèrent à l'action pour défendre la religion populaire. Jacques Roumain fut l'un d'entre eux.

4 Jean Price-Mars affirme que la création du Bureau est due à l'action de Jacques Roumain ${ }^{8}$ sans toutefois expliquer le paradoxe déjà signalé ailleurs (Charlier-Doucet 2001: 59), à savoir, pourquoi le gouvernement de Lescot - celui-là même qui pourchassait férocement les vodouisants et détruisait leurs objets cultuels - est aussi celui qui créa une institution publique pour recueillir et protéger ces objets. La petite histoire évoque le jeu des alliances et des amitiés. Selon celle-ci, derrière Roumain se profile l'ombre de son ami intime et condisciple Kurt Fisher, gendre du sénateur Fombrun, éminence grise sous Lescot $^{9}$. Fisher aurait présenté le projet comme venant de lui pour convaincre et rassurer Lescot que Jacques Roumain, désormais tout à ses activités scientifiques, ne risquait point de nuire au gouvernement ${ }^{10}$. Un autre facteur, qui joua probablement un rôle dans la création du Bureau, est l'intérêt qui existait depuis le xix ${ }^{e}$ siècle pour l'archéologie en Haïti. Cinq ans auparavant, Edmond Mangonès et le Dr Louis Maximilien avaient organisé une exposition dans le but de sensibiliser le public lettré sur les trésors du passé précolombien haïtien. Quoi qu'il en soit, on peut affirmer que la création du Bureau d'ethnologie obéit, à l'origine, à un souci de préservation du patrimoine ethnologique et archéologique haïtien, alors menacé tant par les actions du gouvernement et sa puissante alliée l'Église catholique que par celles de pilleurs et de vandales isolés, chercheurs d'or d'un nouveau genre, qui agissaient en toute impunité.

\section{La mission du Bureau d'ethnologie}

5 Dans le décret-loi du 31 octobre 1941 portant création du Bureau d'ethnologie de la République d'Haïti, celui-ci est conçu comme un établissement de recherche, de vulgarisation et de sauvegarde du patrimoine devant œuvrer dans les domaines de l'ethnographie et de l'archéologie ${ }^{11}$. Cinq tâches lui sont assignées. La première consistant à «inventorier, classer et conserver les pièces ethnographiques et archéologiques » semble faire référence à la collection d'objets provenant directement de la campagne de rejete $^{12}$ et relève davantage d'un musée, dont l'organisation sera d'ailleurs la troisième tâche confiée au Bureau. Les autres tâches prévues en 1941 sont «l'investigation méthodique et la protection des sites archéologiques " (tâche $n^{\circ} 2$ ), «le développement de l'enseignement de l'ethnologie» (tâche $\mathrm{n}^{\circ} 4$ ) et "la publication d'un bulletin comportant le résultat des recherches du Bureau et les travaux d'ethnologues haïtiens et étrangers" (tâche $\mathrm{n}^{\circ}$ 5). Né dans un contexte de persécution du vodou, le Bureau se donna aussi pour tâche immédiate de recueillir les objets témoins du culte, de protéger et défendre les vodouisants, et d'étudier scientifiquement leurs pratiques.

En effet, pour l'équipe de départ (composée de Jacques Roumain, Kurt Fisher, Edmond Mangonès, Louis Maximilien) comme pour les suivantes (Lorimer Denis, Emmanuel C. Paul, Jacques Oriol), il s'agissait de procéder à un inventaire systématique de la culture haïtienne en mettant l'accent sur deux de ses deux composantes ignorées et méprisées jusqu'ici, l'africaine et l'amérindienne. Projet scientifique sous-tendu par un objectif moral et pédagogique à l'intention des élites du pays : faire connaître les coutumes populaires et les faire respecter par l'oligarchie afin que soient combattus les préjugés de toute sorte considérés comme les causes premières du «mal haïtien ». Cela revenait à proposer une nouvelle définition de la culture et de l'homme haïtien, qui prenait le contre-pied de celle prévalente depuis le $\mathrm{XIX}^{\mathrm{e}}$ siècle, selon laquelle Haïti, tout en se 
proclamant fière de ses origines africaines et de son appartenance raciale se vantait d'être en fait une province intellectuelle de la France. Cette attitude paradoxale était celle des élites (noires et mulâtres) qui, s'affirmant seules détentrices des secrets de la " civilisation », s'autoproclamaient représentatives de la «nation». C'est contre cette vision, qui mettait l'accent sur l'élément européen de la culture haïtienne en ignorantles autres, que s'étaient élevés Jean Price-Mars et les indigénistes, et que le Bureau, en s'inspirant des Griots et en s'appuyant sur des recherches empirique, se proposait d'infirmer sur des bases scientifiques.

7 Jacques Roumain ne resta pas assez longtemps à la tête du Bureau pour élaborer en détail les objectifs de l'institution nouvellement créée et la mission de l'ethnologie en Haïti. Ce sont dans les écrits de Lorimer Denis (directeur de 1946 à 1957) et d'Emmanuel C. Paul (directeur de 1957 à 1963) que la vocation du Bureau d'ethnologie va être précisée et définie. Cependant Jacques Roumain nous a laissé un texte où il exprime clairement ce que devait être, à ses yeux, le musée du Bureau d'ethnologie: un espace scientifique et éducatif, « destiné à l'enseignement des couches les plus larges de la population, [dont la] mission n'est pas de séduire une curiosité désœuvrée, mais d'instruire et de stimuler l'étude » (Roumain 1943: 34). Quant à son approche de l'ethnologie comme discipline, on sait que Roumain était un grand admirateur de Jean Price-Mars, qu'il avait accompagné plusieurs fois dans ses recherches à travers la campagne haïtienne ${ }^{13}$. Il est permis de penser que Roumain partageait globalement son approche ethnographique.

\section{Rôle de l'ethnologie dans la société haïtienne}

8 Avant d'aborder la pensée de ce que certains ont appelé « l'école d'ethnologie haïtienne », un coup d'œil sur le contexte sociopolitique de l'époque s'impose. Avec l'élection du président Dumarsais Estimé faisant suite aux mouvements sociaux de 1946, un fait décisif s'est produit : l'accession au pouvoir de la classe moyenne noire, dont le développement, timide auparavant, s'était accéléré depuis l'occupation américaine. Les porte-parole de cette catégorie sociale se disaient les "authentiques" représentants du peuple et revendiquaient le pouvoir en son nom. Apeurée, l'oligarchie renversa le président Estimé et mit à sa place le général Paul E. Magloire pour défendre ses intérêts. Magloire fut renversé à son tour et François Duvalier accéda au pouvoir. Concoctée à partir d'une série de facteurs externes, l'idéologie politique de celui-ci (combinaison de nationalisme, d'indigénisme, de noirisme et d'authentisme), qui portait en germe le totalitarisme, la violence et l'exclusion, aboutit à la dictature tristement célèbre. L'ombre de Duvalier, comme nous le verrons, hanta, et continue de hanter le Bureau d'ethnologie et la perception de la discipline en Haïti.

9 Lorimer Denis, par exemple, est rarement évoqué sans que ne lui soit associé « son frère spirituel », François Duvalier. Nous y reviendrons. Pour le moment, attachons-nous à présenter la vision de Lorimer Denis, et surtout celle d'Emmanuel C. Paul qui peut être considéré comme le maître à penser de cette «école d'ethnologie haïtienne ». Ce fut lui qui écrivit le plus abondamment sur sa conception de l'ethnologie et sur les responsabilités de l'ethnologue noir en général et de l'ethnologue haïtien en particulier. 


\section{La société haïtienne mise en équations}

10 Lorimer Denis a exprimé sa conception de l'ethnologie et du folklore dans plusieurs articles et conférences. Quant à Emmanuel C. Paul, c'est surtout dans deux articles, «L'ethnologie en Haïti » (1949) et "Tâches et responsabilités de l'ethnologie » (1959), qu'il développe le mieux ses idées sur les objectifs de la discipline en Haïti. Pour l'un comme pour l'autre, l'ethnologue haïtien doit faire une œuvre à la fois scientifique et civique, en un mot, ils prônent une « ethnologie engagée ».

11 Dans un premier temps, il fallait défendre la discipline elle-même contre les attaques dont elle était l'objet, en démontrant qu'elle n'était pas une « science spéculative, comme le prétendaient des docteurs et politiciens mal informés ». Pour cela, il était nécessaire de mettre l'accent sur ses applications aux plans politique et pratique. Il est indéniable, argumente Paul, que l'ethnologie peut rendre de grands services en fournissant des informations fiables pour aider aussi bien les gouvernements que les cadres et les techniciens à prendre des décisions qui s'appuient sur une connaissance des pratiques (trop souvent ignorées) de la population locale. Dans cette optique, «le Bureau d'ethnologie se devait d'être le bastion de la connaissance scientifique haïtienne » (Alexis 1963: 26).

12 Mais c'est surtout sur le plan du civisme et de la morale sociale que l'ethnologie trouve son utilité première. Price-Mars (1951) l'avait affirmé au début du siècle, Denis (1950) a entrepris de le prouver en montrant que folklore et patriotisme sont liés - le premier alimentant et fortifiant le second. Pour E. C. Paul (1959 : 18) également, la tâche de l'ethnologue est avant tout patriotique. Elle consiste à « poursuivre sur le plan spirituel l'œuvre de la révolution politique et sociale réalisée par nos aïeux ». En effet, explique-til, le nationalisme haïtien s'est toujours alimenté à deux sources: l'histoire et l'ethnologie. Se référant à la résistance culturelle et politique sous l'occupation américaine, Paul conclut que « l'ethnologie s'est révélée en Haïti une science de combat. Elle nous a permis de nous défendre et de résister à toute tentative d'absorption totale ». Désormais, il faut faire de l'ethnologie, pour construire, pour «travailler à l'édification d'un nationalisme culturel » (ibid.).

13 La «nation » qu'il s'agit de bâtir est définie par les ethnologues de l'époque comme « un Tout fort et cohérent » qui ne peut se concevoir sans une « complète unification morale et spirituelle » (Paul 1949: 36-37). Selon eux, le grand mal haïtien, c'est la dichotomie sociale qui oppose « la partie minoritaire, les élites », aux " masses attardées ». C'est également le mépris social dont sont victimes ces masses elles-mêmes, alors qu'elles sont les véritables dépositaires de «l'âme nationale» (Denis 1950: 43). Et Denis pose l'équation fondamentale de cette école d'ethnologie : ce sont « les extériorisations du peuple » (art, chants, danses) qui constituent véritablement «la spiritualité de la nation haïtienne» (ibid.).

14 Aussi la solution au problème se trouve-t-elle à la fois dans la valorisation de la culture populaire et le changement d'attitudes des élites vis-à-vis de cette dernière. Tout comme Price-Mars, les ethnologues haïtiens de cette période ont prêché un évangile d'union entre les élites et le peuple, union qui passe d'abord par une "refonte de notre mentalité ». Et ils se donnent pour mission d'établir «un pont entre le peuple et les élites ». Car, explique E. C. Paul (1949: 16-17), « lorsque nous décrivons les manifestations de ce que les uns appellent notre gros peuple, les autres, notre petit peuple, l'un de nos 
buts est de mettre en valeur ses qualités eu égard à ses conditions de vie et provoquer en sa faveur un courant de sympathie faite de compréhension ». Ainsi, ce " véritable courant de sympathie en faveur de nos masses attardées nous guérira de cette honte que nous avons de nous-même, enfin, de tout complexe d'infériorité » (ibid.: 37). Dans cette optique, selon la formule de Lorimer Denis (1950), faire de l'ethnologie devient un « apostolat scientifique».

mission rédemptrice est assignée à l'ethnologie dans le monde noir en général. Ce monde encore dominé par le joug colonial doit « conquérir son autonomie sur tous les plans $»^{14}$. Aux ethnologues noirs du monde entier, E. C. Paul propose l'exemple d'Haïti, pays où a eu lieu un " réveil de la conscience nationale sous l'influence de l'ethnologie ». Eux aussi, «en contribuant au développement de leur discipline, doivent en faire une puissance de combat en sorte qu'elle remplisse ses fonctions sociales dans le sens de la revalorisation des cultures négro-africaines $»^{15}$.

16 Cette ethnologie engagée doit être aussi, avant tout, une ethnologie «scientifique ». Pour E. C. Paul, il n'y a pas de contradiction entre les deux. Il revendique pour les ethnologues noirs le droit d'étudier leur propre culture sans être taxés de partialité, à un moment où l'anthropologie euro-américaine est considérée comme la seule à pouvoir porter un regard objectif sur les cultures des « Autres ».

17 Après cette époque fondatrice qui s'achève avec J. Oriol, lequel partage pleinement la vision des pionniers ${ }^{16}$ (faire connaitre pour corriger), on ne trouvera plus de réflexion sur le rôle du Bureau. Quant à la place de l'ethnologie en général, il faudra attendre les années 1980 pour que s'amorce, avec Max Paul, une réflexion théorique sur la problématique du développement culturel dans le cadre de la mondialisation.

Cette mission une fois définie, comment s'est effectuée la mise en œuvre? Quels ont été les programmes de recherche et les champs d'intérêt du Bureau?

\section{Les projets de recherche et les publications}

Pour dégager les grandes orientations de recherche du Bureau ${ }^{17}$, nous nous appuierons sur une analyse des titres publiés pendant deux grandes périodes 1941-1963 et 1963-2003. La fréquence de ces publications servira d'indicateur des activités du Bureau, avec une réserve cependant. Il faut, en effet, souligner que ces données sont provisoires, car, faute de fonds, toutes les recherches effectuées par le Bureau n'ont pu encore être publiées, et un grand nombre de notes manuscrites - certaines remontant aux premières années d'existence du Bureau - attendent toujours d'être mises à la disposition des chercheurs.

Pour la première période de vingt-deux ans (1941-1963), la production totale est de vingt publications ${ }^{18}$. Il y a eu deux moments saillants : 1942-1943, avec cinq publications en deux ans, et 1958, année pendant laquelle le Bureau a produit trois publications. Autrement, la moyenne est d'une publication par an, parfois deux. Il y eut quatre années d'interruption $(1945-1946$; 1954; 1957), périodes qu'il serait intéressant de relier au contexte économique - marasme - et politique - périodes électorales. En 1958 s'instaure la pratique de rassembler trois numéros en une seule publication; cette fréquence fut maintenue pendant trois ans, puis en 1960, ce fut à nouveau un numéro par an.

21 Pour la période 1963-2003, soit quarante ans, le nombre de publications se chiffre à quinze. On peut distinguer une période de vingt ans, 1963-1983, pendant laquelle le Bureau doit faire face à des difficultés, comme en témoigne le nombre de publications et 
surtout, par les fréquentes et longues interruptions de parution du Bulletin. Il s'agit de la période des Duvalier (père et fils). Une reprise semble s'amorcer entre 1984-1986 (fin du gouvernement de Jean-Claude Duvalier) : le Bureau publie cinq bulletins en trois ans. De 1987 à 2003 (soit seize ans), il fait paraitre quatre publications, la dernière remontant à 1998. On est donc passé de trois publications par an, entre 1941-1943 (et ce, en dépit de l'impact négatif de la Seconde Guerre mondiale sur l'économie haïtienne) ${ }^{19}$, à une seule réunissant plusieurs numéros (1958-1960), pour aboutir à la situation actuelle d'une seule livraison couvrant deux, voire même trois ou cinq années.

Le nombre total de publications est certes très bas. Mais placés dans les contextes économiques et politiques respectifs, ces chiffres permettent de comprendre à quel point les aléas de la conjoncture nationale et internationale ont eu une influence négative sur le développement du Bureau.

\section{Les domaines de recherche}

Les recherches menées par le Bureau se sont orientées en fonction des différentes tâches définies par le décret de fondation. Dans un premier temps, il s'est consacré à la fois à l'étude de l'archéologie précolombienne et du vodou. Mais très vite les recherches ethnographiques se sont étendues à d'autres domaines de la culture populaire.

Des recherches archéologiques furent menées en Haïti dès le XIX ${ }^{\mathrm{e}}$ siècle, surtout par des étrangers, archéologues européens d'abord (de 1804 à 1825) puis américains (de 1851 à 1939) ${ }^{20}$. Ainsi, au début $d u x^{e}$ siècle, les institutions culturelles nord-américaines, stimulées par la Société des Américanistes de Paris, lancèrent un vaste projet de recherches archéologiques visant une synthèse continentale (cf. Mangonès \& Maximilien 1941: 5). C'est avec la création du Bureau en 1941 que, pour la première fois, des recherches furent entreprises sous les auspices d'une institution culturelle haïtienne. Le Bureau signa un accord avec le musée Peabody de l'université de Yale qui, dès 1936, avait lancé un programme de recherches caraïbéennes. Cependant, l'implication du Bureau demeura très limitée, car il ne disposait ni des ressources matérielles ni des ressources humaines pour jouer un rôle substantiel et décider des programmes de recherche. Ses membres y trouvèrent cependant leur compte. Ainsi, pour l'un des pionniers de l'archéologie haïtienne, le Dr Louis Maximilien, l'intérêt scientifique était lié à un désir d'action sociale : dans ce domaine aussi il fallait changer les préjugés, notamment sur les Amérindiens, préjugés qu'avaient intériorisés la plupart des lettrés haïtiens. En effet, à de rares exceptions près, les populations précolombiennes étaient vues à travers le prisme des relations des chroniqueurs européens et des manuels d'histoire à l'usage des écoliers haïtiens qui, les uns et les autres, véhiculaient des stéréotypes sur les Indiens (race inférieure, couardise, indolence, culture primitive, etc.) qu'il convenait de redresser ${ }^{21}$. Le Bureau voulait prouver l'existence de survivances amérindiennes - jusqu'alors réputées quasi inexistantes dans la culture haïtienne - et étudier les éléments de syncrétisme amérindien-africain qui s'étaient surtout développés dans l'espace religieux du vodou.

Une analyse sommaire des titres publiés pendant la période 1941-1963 révèle que si, au début des années 1940, l'archéologie et le vodou dominent, au cours des années 1950, les champs d'intérêt vont en s'élargissant. En plus du folklore, les thèmes reflètent les préoccupations du moment et les débats en cours dans la société haïtienne. Le Bureau a affirmé sa position avant-gardiste sur deux sujets alors très controversés : la nécessité d'haïtianisation de l'éducation qui doit s'inspirer des coutumes locales (Paul 1956), et la 
valorisation du créole qui doit être considéré comme une langue à part entière et être utilisé dans l'éducation populaire ${ }^{22}$. Sur la santé mentale, domaine à la fois tabou et négligé, le Bureau, par la voix de Louis Mars (1947) plaide, d'une part, pour un meilleur traitement des malades mentaux et, d'autre part, pour l'ouverture du premier centre de psychiatrie en Haïti.

Entre 1955 et 1958, les échanges avec l'extérieur s'intensifient. Dans les publications du Bureau, se manifeste une ouverture vers l'Afrique, la Caraïbe et le Brésil, et l'approche comparatiste fait son apparition avec Roger Bastide (sur les complexes afro-brésiliens), Jacques Lombard (sur les chefferies administratives d'Allada), Melville J. Herskovits (contes populaires dahoméens), George E. Simpson (comparaison de la religion en Haïti et à la Jamaïque). Les thèmes se rapportant à Haïti dans les Bulletins coïncident plus étroitement avec les centres d'intérêts de chercheurs étrangers - surtout nordaméricains, mais aussi européens - en quête de survivances africaines dans la Caraïbe ou sur le continent américain. Les sujets traités - la famille, l'organisation sociale, le travail collectif et, bien sûr, le vodou - sont le reflet des domaines qui intéressent cette communauté scientifique étrangère. La question de l'économie fait son apparition avec l'étude de Sidney Mintz (1960) sur les marchés ruraux ${ }^{23}$. Durant la décennie 1960-1970, les publications du Bureau sont centrées sur la culture matérielle des couches populaires (poterie, vannerie) et sur la musique (étude d'organographie haïtienne). Les travaux des années 1980 manifestent un regain d'intérêt pour l'archéologie, l'histoire et la linguistique. Les phénomènes religieux, quant à eux, font l'objet d'un retour sur le devant de la scène scientifique dans les années 1990, c'est le cas du vodou, mais également des phénomènes charismatiques protestants et catholiques.

Ce tour d'horizon permet de se rendre compte que les publications du Bureau, bien que centrées sur la problématique haïtienne - et c'est là leur apport le plus important témoignent aussi d'une thématique variée, qui coïncide avec les grandes orientations des travaux ethnographiques des centres de recherche occidentaux. Quant à la qualité des publications elles-mêmes, si on s'en tient aux jugements d'universitaires étrangers, on peut affirmer que celles produites dans les années 1940-1950 ont été favorablement accueillies par la communauté scientifique, aussi bien en Europe, aux États-Unis que dans la Caraïbe. Comme l'écrit Roger Bastide, Haïti, à l'époque, « s'est placée au premier rang des études d'africologie sur le continent». Les témoignages de Jacques Butterlin, Alejo Carpentier, Aimé Césaire, Michel Leiris, Alfred Métraux, André Schaeffner, pour ne citer que ceux-là, vont dans le même sens.

\section{Histoire institutionnelle}

À l'origine, le décret-loi de création (1941) précise seulement les cinq tâches du Bureau. Aucune mention de financement ni de structure administrative, aucune disposition concrète n'est prise par le gouvernement pour assurer son fonctionnement. Le Bureau n'a jamais obtenu le budget qu'il aurait dû avoir pour mettre en œuvre les tâches qui lui étaient assignées. Il en découle une situation financière plus que précaire. Et ce sera une constante dans son histoire. En comparaison avec les autres institutions culturelles publiques du pays, le Bureau d'ethnologie a toujours fait figure de parent pauvre et cela continue aujourd'hui. Les difficultés que rencontre cette institution sont constamment évoquées par les directeurs et par ses membres, des origines à nos jours, difficultés dont divers bulletins font état et qui témoignent d'un manque d'intérêt certain de la part des 
pouvoirs publics pour cette institution. Les gouvernements successifs se sont contentés de soutenir le Bureau à l'aide de subsides ou d'allocations que certains fonctionnaires bienveillants lui octroyaient, pour la plupart d'anciens membres du Bureau ayant abandonné l'ethnologie et occupant un poste politique important ou une haute fonction dans l'administration publique (Edmond Mangonès, Léonce Viaud, Michel Lamartinière Honorat, par exemple). Des allocations provenaient aussi plus ou moins régulièrement de la mairie de Port-au-Prince ou d'autres institutions de l'État (loterie de l'État haitien, ministères de l'Intérieur, de l'Éducation nationale, etc.) au gré des fonctionnaires en place. Et, durant les premières années de son existence, le Bureau a également fonctionné sur la base de dons de particuliers, le plus souvent amis et connaissances des membres du Bureau, dons qui sont devenus de plus en plus rares par la suite.

En 1982, dans la foulée de la réforme administrative exigée du gouvernement de JeanClaude Duvalier par les instances financières internationales, il y a eu une tentative de réorganisation structurelle de la fonction publique haïtienne. Michelle Bennett Duvalier, pour des raisons qui restent à déterminer, manifesta un certain intérêt pour les arts en Haïti. À son instigation est créé l'Institut national haïtien de la culture et des arts (Inhaca) qui regroupe l'ensemble des institutions culturelles publiques.

L'appellation du Bureau change alors et devient le Bureau national d'ethnologie. Sa structure administrative et son organisation sont spécifiées dans un nouveau décret (24 octobre 1984). Désormais, il émarge au budget de la République à travers l'Inhaca. Des projets de restructuration, de rénovation s'élaborent et surtout celui de transformer le musée du Bureau (fermé vers la fin des années 1970) qui doit renaitre sous l'appellation pompeuse de Musée de l'Homme haïtien. Dans la pratique, rien n'a changé. Les quatre sections principales, établies dès 1941 (musée, bibliothèque et les deux sections techniques: archéologie et ethnologie), continuent d'exister, du moins nominalement. Après le renversement de J.-C. Duvalier et la disparition subséquente de l'Inhaca en 1986, le Bureau est placé sous la tutelle du ministère de l'Éducation nationale, puis du ministère de la Culture nouvellement créé.

31 Malgré les nouvelles dispositions et les changements de tutelle, la situation générale du Bureau, entre 1982 et 2003, ne connaît qu'une amélioration minime. L'attribution d'une enveloppe budgétaire nettement insuffisante contribue à prolonger une situation financière précaire. Par ailleurs, le mécanisme de décaissement des fonds alloués au Bureau consacre sa dépendance vis-à-vis des pouvoirs politiques, lesquels déterminent les "priorités budgétaires» au gré du programme politique du moment. De fait, une lourdeur administrative paralyse toute velléité d'activités et le Bureau souffre, lui aussi, des maux et dysfonctionnements de toute l'administration publique du pays.

$\mathrm{Au} v \mathrm{vu}$ de ces difficultés financières qui ont constamment traversé son histoire, on pourrait même s'étonner que le Bureau soit parvenu à mener quelques activités importantes et à produire des publications d'un certain niveau, même en nombre restreint et de valeur inégale, il est vrai. On comprend que les ressources locales n'aient jamais permis de lancer de grandes campagnes de fouilles archéologiques, généralement très coûteuses, et que le Bureau ait plutôt mis l'accent sur les recherches ethnographiques, relativement moins onéreuses et demandant un matériel moins sophistiqué.

33 L'une des stratégies adoptées dès le début pour pouvoir mener des recherches a été de s'associer à des institutions étrangères (universités, musées) qui finançaient elles-mêmes 
leurs activités en Haïti. Le Bureau offrait certaines ressources humaines et sa connaissance du terrain, mais ne recevait pas de fonds. Les résultats des recherches et les analyses des chercheurs étrangers étaient discutés la plupart du temps avec les membres du Bureau, mais celui-ci n'avait pas droit de regard sur les publications. Jusqu'à l'administration de Jacques Oriol (1963-1983), le Bureau s'est farouchement refusé à recevoir des fonds de l'étranger pour son fonctionnement - cela aurait signifié « inféoder l'État à des étrangers ", répétait ce dernier, et ce serait dévier du "nationalisme économique » professé par les aînés. Sous la direction de Max Paul (1983-1991), le Bureau s'est ouvert à l'aide financière internationale, laquelle est restée minime (dons occasionnels de l'ambassade d'Allemagne et de l'Organisation des États américains). La crise persistante depuis 1991 interdit de penser à tout apport de fonds substantiels.

Le manque de ressources humaines fait aussi partie des "difficultés de tous ordres » auxquelles le Bureau est confronté, depuis ses origines. Cet organisme a toujours fonctionné avec un personnel administratif et technique insuffisant par rapport à ses besoins. Par exemple, à la mort de E. C. Paul, il n'y avait que trois ethnologues attachés au Bureau. Mais ce qui semble avoir changé au fil du temps, c'est avant tout le degré d'investissement personnel des membres dans ses activités : au feu sacré des pionniers semble avoir succédé un certain désabusement et un sentiment d'impuissance des dirigeants et des membres qui leur ont succédé.

La première équipe des responsables du Bureau réunit des hommes d'origine sociale différente (haute bourgeoisie mulâtre, petite-bourgeoisie et classe moyenne noires), se réclamant tous des tendances gauchisantes de l'époque. À cette équipe sont venus s'adjoindre des intellectuels haïtiens renommés, eux aussi issus de milieux sociaux divers, pour donner des conférences ou participer à d'autres activités académiques. On peut citer en particulier des membres de la Société haïtienne d'histoire et de géographie, tels Jean Price-Mars, Catts Pressoir, Pradel Pompilus, Louis Mars. Par la suite, à part quelques fidèles, tels Louis Mars ou Pradel Pompilus, le Bureau ne semble plus avoir bénéficié du support de l'intelligentsia haïtienne.

À l'époque des pionniers, le Bureau, fidèle à la tâche d'enseignement de l'ethnologie qui lui était assignée, prenait aussi en charge la formation sur le terrain du personnel technique. Comme assistants, Roumain fit ainsi appel à des jeunes étudiants recrutés dans la première promotion de l'Institut d'ethnologie, créé en 1941 par Jean Price-Mars. En retour, ces jeunes offraient leurs services sur la base d'un quasi-bénévolat. Beaucoup d'entre eux ont participé à des recherches avec des ethnologues et des archéologues étrangers de renom ${ }^{24}$ et ont bénéficié de bourses pour parfaire leurs études dans des universités étrangères. Sous la direction d'Emmanuel C. Paul, cette tradition d'encadrement des jeunes ethnologues par le Bureau s'est pérennisée ; et, en dépit des maigres ressources de l'Institut d'ethnologie, son directeur allouait des bourses à quelques étudiants et encadrait leurs travaux de terrain. Ces pionniers avaient embrassé la profession d'ethnologue " par vocation », pour reprendre les mots d'Emmanuel C. Paul et Jacques Oriol. Ce sont aussi eux qui ont le plus produit dans leur jeunesse. Ensuite, soit les aléas de la vie, soit une nouvelle carrière (dans la politique bien souvent), soit la mort les ont écartés de la profession.

37 À partir des années 1980, la carence en personnel technique se fait durement sentir. En outre, le mode de désignation des directeurs change. Dans la première période (1941-1983), trois directeurs sont décédés pendant leur mandat ${ }^{25}$, et leurs successeurs étaient issus des rangs du Bureau lui-même par une sorte de mécanisme interne où l'on 
semblait se " passer le flambeau » pour continuer une « mission». Or, à partir de 1983, le directeur du Bureau est nommé directement par le président de la République et n'est plus nécessairement un ex-membre du Bureau. Il faut cependant souligner que, même si les négociations politiques semblent prendre le pas sur les critères techniques, tous les directeurs entre 1983 et 2003 ont eu une formation en ethnologie.

Quant au personnel technique à proprement parler, il dépasse rarement quatre membres. La politique d'encadrement de jeunes ethnologues, réamorcée dans les années 1980, n'a pas pu se poursuivre, principalement pour des raisons d'ordre financier. Par ailleurs, il faut aussi noter le changement de la population étudiante de la Faculté d'ethnologie, où, depuis les dernières décennies, rares sont les étudiants qui souhaitent faire carrière dans cette discipline. Aujourd'hui, ethnologues et anthropologues de profession sont plutôt rares en Haïti. Et le Bureau d'ethnologie ne trouve plus à recruter dans son vivier naturel ${ }^{26}$, la Faculté d'ethnologie.

39 En se fondant sur les informations publiées dans les Bulletins du Bureau, on peut distinguer, comme on l'a fait pour les publications, une période d'activités relativement intenses (1941-1963), puis un ralentissement (1963-1983), un début de relance (1983-1986) suivi d'une stagnation depuis 1987. Les différentes conjonctures sociopolitiques et économiques (locales et internationales) peuvent expliquer ces mouvements, mais il ne faudrait pas ignorer les facteurs liés aux motivations personnelles.

40 En effet, durant les vingt-deux premières années (1941-1963), il semble régner au Bureau une atmosphère d'activité intense, comme en témoignent les bulletins de cette période. Le Bureau, à cette époque, joue le rôle de catalyseur pour les manifestations à caractère populaire. Il organise de nombreux galas folkloriques avec ses propres danseurs et ses deux chœurs de chants traditionnels, le chœur Mater Dolorosa (chœur populaire vodou ) et le Chœur des Jeunes Filles en fleur. Il apporte une aide technique à de nombreuses organisations culturelles populaires ou d'inspiration plus savante. Il a aussi été à l'origine de la création de la Troupe folklorique nationale, du Musée du Peuple haïtien, du Théâtre de Verdure; il a participé à l'organisation des grands événements culturels d'alors et donné des consultations techniques (célébration du bicentenaire de Port-au-Prince en 1949-1950, et du tricinquantenaire de l'Indépendance d'Haïti en 1954, participation d'Haïti au Festival des Arts Nègres de Dakar). Il maintient aussi des liens avec les organismes officiels chargés de développer le tourisme. Au plan de l'archéologie, le Bureau a suscité et a alimenté l'intérêt du public, ce qui s'est traduit par de nombreux dons provenant de tous les coins de la République, en même temps que la constitution de collections privées d'une certaine importance.

41 D'un point de vue pédagogique, le Bureau mène également de nombreuses activités : il offre des cours aux étudiants des écoles normales rurales et urbaines, et à ceux de la Faculté de médecine, et des cycles de conférences à l'intention d'un plus large public, et ce, à un rythme soutenu (en 1952, le Bureau donne une conférence par quinzaine). Il organise aussi des cours du soir et d'alphabétisation. Il participe à des collectes de fonds pour des organismes à caractère social (Ligue contre le cancer, Croix-Rouge, Centre psychiatrique, etc.). En conséquence, à cette époque, le Bureau est très visible sur les scènes artistique, culturelle et sociale du pays, tant dans la capitale qu'en province.

Sur le plan international, le Bureau, pendant cette période, maintient des liens et des échanges avec de nombreuses institutions scientifiques et culturelles étrangères. Ses membres sont invités à participer à de nombreuses manifestations scientifiques, conférences ou congrès aux États-Unis, en Europe, en Afrique et en Amérique du Sud. Des 
personnalités du monde intellectuel et artistique visitent le Bureau, sans compter les chercheurs étrangers avec lesquels des rapports réguliers sont maintenus.

En dépit de ces actions, l'acceptation de la mission du Bureau est plutôt lente dans la société haïtienne. En 1952, par exemple, malgré certains succès connus à l'extérieur, la lutte pour la reconnaissance de la culture populaire est encore difficile sur le plan local comme en témoigne Léonce Viaud (1952) : « Malgré l'incompréhension du milieu, malgré l'indifférence des uns et des autres, notre institution tient bon et accomplit avec patience et ferveur la mission pour laquelle elle a été créée.»

Pendant les quarante années suivantes (1964-2003), les activités du Bureau, à part de rares moments de sursaut, sont pratiquement restées au point mort.

Peut-on tenter une évaluation critique des apports du Bureau sur le plan intellectuel, institutionnel et social ? À l'anthropologie générale, l'ethnologie haïtienne a apporté deux innovations conceptuelles. La première eut certes un certain écho à l'époque (Alexis 1963: 18), mais pas de résultats immédiats, du moins dans les milieux occidentaux. Il s'agit du plaidoyer de E. C. Paul au Premier congrès des artistes et écrivains noirs de Paris (1956), dans lequel, rejetant l'argument de la plus grande objectivité des spécialistes étrangers, il revendiqua le droit pour les chercheurs des pays non industrialisés d'étudier eux-mêmes leur propre culture. C'était lancer l'idée d'une " anthropologie native» avant la lettre, près de trente ans avant que l'idée n'ait fait son chemin dans le monde intellectuel occidental, à partir des questionnements et critiques postmodernistes des années 1970-1980. La seconde innovation fut incorporée dans le bagage conceptuel de la communauté scientifique mondiale : c'est le concept d'ethnopsychiatrie ou de psychiatrie transculturelle apportée par Louis Mars ${ }^{27}$.

Le Bureau d'ethnologie contribua aussi grandement à la connaissance de la société haïtienne et surtout de la culture populaire, qu'il avait entrepris d'inventorier de manière systématique. Cependant, la vision partagée par le Bureau et les différents courants indigénistes de l'époque prônant une « haïtianité authentique » qui se trouverait à l'état "pur» dans les couches populaires rurales, celle d'une "âme nationale innée et immuable ", et surtout le fait de poser un déterminisme culturel et racial comme base de leur analyse sociale, se révèlent être des prémisses erronées ${ }^{28}$. Et les récupérations idéologiques auxquelles cette vision a donné lieu ont contribué à enfermer davantage dans leur misère les masses dont précisément les ethnologues du Bureau souhaitaient l'avancement.

47 Sur le plan institutionnel, le Bureau a été créé pour être le premier centre public de recherches du pays dans les domaines de l'ethnologie et de l'archéologie. Si, au fil des ans, comme nous l'avons vu, il s'est acquitté de ces tâches avec plus ou moins de succès, le rôle de "protection» des sites archéologiques qui lui avait été dévolu en 1941 n'a, en revanche, jamais pu être assumé. Aujourd'hui, une nouvelle institution publique, l'Institut de sauvegarde du patrimoine national (Ispan) a en charge cette protection. Créé en 1979, cet organisme, à cause du manque de moyens, est lui aussi impuissant à arrêter le pillage et la destruction irrémédiable du patrimoine archéologique haïtien (terrestre et maritime), en dépit de certaines dispositions légales, malheureusement incomplètes.

Le Bureau d'ethnologie reste encore le seul établissement public de recherches dans le domaine de l'ethnographie, du moins nominalement. Une autre institution sœur, l'Institut d'ethnologie, créé en 1941 et devenu Faculté d'ethnologie depuis 1979, a plutôt vocation d'enseignement. Durant les dernières décennies, on a assisté à l'émergence 
d'institutions privées de recherches en sciences sociales qui ont mené des études en collaboration avec des organismes étrangers. Alors que certaines d'entre elles ont signé d'importants contrats avec des institutions internationales pour des recherches sur la culture haïtienne (Romain 1984), le Bureau, lui, n'a pas été sollicité, signe de l'inintérêt dont il est l'objet.

Il est indéniable qu'à ses débuts, en dépit d'une certaine résistance venant en majorité de l'oligarchie catholique ${ }^{29}$, et parfois du gouvernement lui-même ${ }^{30}$, le Bureau a pu exercer une influence positive sur la promotion de la culture populaire, du moins dans la sphère du tourisme : galas folkloriques, chants et danses traditionnels dans les fêtes publiques et dans les hôtels sont aujourd'hui monnaie courante. Il a surtout stimulé la réflexion et initié des changements dans les domaines de l'éducation, de la langue et de la religion. Cependant soixante ans après, les débats qui continuent d'agiter la société haïtienne sur la culture populaire, notamment sur le créole et le vodou, prouvent que le Bureau est loin d'avoir atteint l'objectif qu'il s'était fixé : « Faire connaître et respecter ».

Aujourd'hui, la décadence du Bureau, amorcée il y a quarante ans, semble atteindre un point critique. Il est oublié de la société et surtout délaissé par les pouvoirs publics. Rares sont ceux qui connaissent son existence et, pour ceux-là, le Bureau, confondu avec la Faculté d'ethnologie, est perçu négativement ${ }^{31}$. On peut avancer, sans risque de se tromper, que l'histoire politique récente a considérablement desservi le Bureau. Et là, il faut évoquer la figure de François Duvalier. Le long règne des Duvalier - père et fils -, ne fut pas sans laisser de traces sur l'image du Bureau et sur le développement de l'ethnologie en général. Les liens réels et supposés de François Duvalier avec l'ethnologie, le Bureau et la Faculté d'ethnologie, restent à examiner rigoureusement. Il n'en demeure pas moins qu'on ne saurait parler de l'ethnologie en Haïti sans mentionner François Duvalier.

51 Tout d'abord, il y a l'étroite association entre François Duvalier et Lorimer Denis, qui étaient considérés comme deux "frères spirituels " (Oriol 1958: 11). En outre, de 1946, accession de Lorimer Denis à la tête du Bureau, à 1971, mort de François Duvalier, ce dernier en était Directeur honoraire en titre. Cependant, des témoignages concordants affirment que ce titre ne correspondait à aucun intérêt réel de Duvalier pour l'institution, qu'il ne fréquenta guère. Au contraire, il est à noter que Duvalier négligea le Bureau, alors même qu'il exploitait sa connaissance de la culture populaire et en particulier du vodou, pour se maintenir au pouvoir ${ }^{32}$. Des deux hommes, rapportent leurs contemporains, l'ethnologue c'était Lorimer Denis. Duvalier aurait exploité l'envergure de ce dernier pour se donner une figure de savant, et les articles, bien que co-signés, étaient surtout l'œuvre de Denis ${ }^{33}$.

52 D'un autre côté, il y a aussi le fait que certains membres du Bureau d'ethnologie occupèrent des fonctions électives ou des postes politiques dans le gouvernement des Duvalier $^{34}$; certains avaient des liens familiaux avec de puissants personnages réputés être au service du duvaliérisme ${ }^{35}$. Cela revient-il à dire que le Bureau d'ethnologie et la Faculté d'ethnologie ont été des instruments consentants de la politique duvaliériste? Il est à souhaiter qu'une étude rigoureuse de la question soit entreprise par des historiens ou d'autres chercheurs qui apporteront la lumière sur ces faits ${ }^{36}$.

53 Quant aux pouvoirs publics, leur inaction témoigne de leur profond désintérêt pour le développement de l'institution. L'exemple des locaux (errance et indigence) suffira à illustrer ce point. Après soixante-deux ans d'existence, le Bureau national d'ethnologie ni n'occupe un lieu décent ni ne jouit d'un espace qui lui soit propre. À ses débuts, le Bureau 
était logé dans le sous-sol de l'Hôtel de Ville, espace que lui avait octroyé le maire d'alors, Edmond Mangonès. Le Bureau et le Musée y restèrent pendant près de dix ans, de 1941 à 1950. Ensuite, entre 1951 et 1959, ils purent s'installer dans deux anciens pavillons de l'Exposition du Bicentenaire, d'abord l'ancien pavillon des Meubles et Ameublement, puis le local du Musée du Peuple haïtien. Ce Musée, monté en six semaines et dont la collection provenait en grande partie de celle du Bureau d'ethnologie, a été fermé purement et simplement peu de temps après sa création. En 1959, l'ancien local du Musée national (lui-même transféré dans la villa de l'ex-président Magloire) est donné au Bureau. C'est l'espace qu'il occupe depuis quarante-quatre ans et, bien qu'insuffisant, c'est celui que le Bureau a fini par considérer comme sien : une unique salle d'environ $50 \mathrm{~m}^{2}$, divisée en un espace pour le musée et un autre pour la bibliothèque. Abandonné des pouvoirs publics, l'édifice, qui n'a jamais bénéficié de travaux d'entretien, ni de réparations majeures depuis son inauguration en 1938, menace de tomber en ruine ${ }^{37}$.

S'il est souvent vrai que les dates symboles de l'histoire nationale provoquent un sursaut des gouvernements, d'où résultent parfois une création ou une rénovation d'institutions, il serait souhaitable que le Bureau d'ethnologie soit l'objet de leur attention et puisse connaître un renouveau. En 1946 le Bureau d'ethnologie laissa passer l'occasion de retrouver une certaine vitalité grâce aux activités de commémoration du Bicentenaire de Port-au-Prince ; il en sortit même affaibli. En effet, le gouvernement d'Estimé, au lieu de renforcer le Bureau, privilégia la création du Musée du Peuple haïtien. En 1954, les commémorations du Tri-cinquantenaire de l'Indépendance d'Haïti furent une nouvelle occasion ratée. En effet, le gouvernement de Magloire, exploitant à fond la culture populaire comme denrée commerciale et touristique, mit l'accent sur la structuration de l'office du tourisme. Une fois de plus, le Bureau d'ethnologie fut oublié. Le sera-t-il lors de la célébration du Bicentenaire de l'Indépendance d'Haïti, en 2004 ? Ironie de l'histoire qui se répète, des informations vérifiées indiquent qu'il sera à nouveau déplacé et que le local qu'il occupe actuellement, enfin rénové, abritera un nouveau musée créé seulement pour la circonstance.

Aujourd'hui le Bureau d'ethnologie d'Haïti est à l'agonie. Lui fera-t-on une belle oraison funèbre ou bien s'éteindra-t-il dans l'indifférence générale ? L'œuvre créée en 1941 est en train de sombrer dans l'oubli, ou pire, dans une méconnaissance accusatrice. Cette institution sexagénaire, qui a connu des moments de vie intenses et productifs, n'a jamais fait l'unanimité, et pour cause. Associée comme elle l'a été aux idéologies qui ont traversé la société haïtienne (au communisme, à la gauche, à l'anticléricalisme, à la défense du vodou et de la culture populaire), sa présence et son passé dérangent certains secteurs conservateurs de la société haïtienne. D'un autre côté, l'ombre des Duvalier et de leurs Tontons macoutes hante aussi le Bureau, qui est aujourd'hui identifié à l'extrême droite. La récupération de sa vision raciale et essentialiste de la culture et les dérives idéologiques qui s'en sont suivies entachent une institution qui, à tort ou à raison, est perçue par beaucoup, comme un «bastion du duvaliérisme». Dans un contexte où les positions idéologiques se radicalisent et où les sensibilités politiques sont à fleur de peau, on ne peut que souhaiter qu'une analyse approfondie et objective, fondée sur des données dûment vérifiées, soit faite des rapports entre le Bureau et les pouvoirs politiques. Pour l'anthropologie et pour l'histoire, les générations d'aujourd'hui et celles de demain méritent que la vérité soit faite sur le Bureau d'ethnologie d'Haïti et sur son institution sœur, la Faculté d'ethnologie. 
«Votre livre est une précieuse documentation... J'admire le travail fait par le Bureau d'Ethnologie d'Haïti qui a su réunir toute une pléiade de savants et qui se met au premier rang, en Amérique, dans les études d'africologie. », in Bulletin, avril 1952, n $^{\circ}$, p. 50.

«J'ai lu votre précieux essai d'organographie haïtienne. J'ai déjà signalé celui-ci dans la bibliographie de L'Année sociologique et je compte l'utiliser dans mes travaux personnels. », in Bulletin, avril 1952, n 9, p. 50.

"L'ensemble de ces notices apportent une docu-mentation riche et vivante sur le culte vodou en même temps qu'une contribution appréciable à l'étude de la littérature orale de langue créole. ", in Bulletin, avril 1952, n 9, p. 110.

"Je viens de parcourir votre publication et, soit dit sans flatterie, j'ai été extrêmement intéressé par sa lecture. Sans vains discours, vous avez accumulé en peu de pages une somme de renseignements de premier ordre et j'apprécie particulièrement la clarté et la concision de l'exposé. Je vous adresse mes très sincères félicitations et serai toujours heureux de lire vos écrits. "

"Cet ouvrage passe en revue, comme le titre l'indique, les principaux folkloristes et les diverses institutions folkloriques du monde. Outres les photographies de MM. Lorimer Denis et Emmanuel C. Paul qu'il publie, le livre relate les bio-bibliographies des personnalités intellectuelles suivantes de notre milieu : Dr Jean Price-Mars, Dr Francois Duvalier, Me Kléber Georges Jacob, Jacques Roumain, Milo Marcelin, Michel Lamartinière Honorat, Jacques Oriol, Léonce Viaud et Michel Aubourg. ", in Bulletin, avril 1952, n 9, p. 108.

« Je tiens à vous dire également la haute estime dans laquelle je tiens l'activité du Bureau d'Ethnologie. En recueillant et en sauvant de l'oubli ou de l'indifférence des textes folkloriques le Bureau est en train de sauver un des trésors haïtiens les plus précieux. Vous rendez service à la science ethnographique. Vous rendez service à l'art et à la poésie. ", in « Activités du Bureau d'Ethnologie », rapportées par Léonce Viaud, in Bulletin, avril 1952, n 9, pp. 97-114.

« Parallèlement au roman, l'ethnographie connaît en Haïti, un développement réellement prodigieux, si on retient que son opportune orientation, se consacrant à la recherche de la vérité, eut à se heurter, dès le début, à d'inévitables préjugés. À l'ombre du maitre Price-Mars, à qui nous devons l'ouverture des premiers sentiers dans l'exubérante broussaille du syncrétisme religieux haïtien, travaille une équipe de jeunes intellectuels groupés dans cet admirable Musée d'Ethnologie d'Haïti - un des lieux les plus fascinants qui existent dans le continent - dont la conservation est confiée, depuis quelques années, à ce passionné étudiant des religions populaires de son pays qu'est Lorimer Denis. »

\section{BIBLIOGRAPHIE}

ALEXIS, Gerson

1963 « In Memoriam Emmanuel C. Paul », Bulletin du Bureau d'ethnologie de la République d'Haïti, IV-29: 11-29.

ARNoux, Charles

1964 « Index bibliographique des Bulletins et Publications du Bureau d'ethnologie... », Bulletin du Bureau d'ethnologie, IV-30: 47-56.

AUBOURG, Michel 
1951 Haïti préhistorique. Mémoire sur les cultures précolombiennes, ciboney et taïno. Port-au-Prince, Imprimerie de l'État (« Publication du Bureau d'ethnologie de la République d'Haïti » Série II-8).

BASTIEN, Rémy

1944 « Rapport annuel du Bureau d'ethnologie de la République d'Haïti (1942-1943 ) », Bulletin du Bureau d'ethnologie, 3: 1-15.

CHARLES, Arnoux

1964 « Index bibliographique des Bulletins et Publications du Bureau d'ethnologie de la République d'Haïti », Bulletin du Bureau d'ethnologie, IV-30: 47-56.

CHARLIER-DOUCET, Rachelle

2001 « Musées en Haïti », Conjonction, 206: 57-73.

CORVINGTON, Georges

1991 Port-au-Prince au cours des ans. VII. La ville contemporaine: 1934-1950. Port-au-Prince, Imprimerie Deschamps.

DENIS, Lorimer

1950 Quelques aspects de notre folklore musical. Port-au-Prince, Imprimerie de l'État (« Publication du Bureau d'ethnologie de la République d'Haïti » Série II-7).

DORMILUS, Arnold

1995-1997 Répertoire des Bulletins du Bureau national d'Ethnologie. Numéro spécial.

GAILLARD, Roger

1993 « L’indigénisme haïtien et ses avatars », Conjonction, 197: 9-26.

HONORAT, Jean-Jacques

1974 Enquête sur le développement. Port-au-Prince, Imprimerie Centrale.

HONORAT, Michel Lamartinière

1955 « Les danses folkloriques haïtiennes », Bulletin du Bureau d'ethnologie, II-11.

LAGUERRE, Michel S.

1982 Urban Life in the Caribbean. Cambridge, MA, Schenkman.

MANGONÈs, Edmond \& MAXIMILIEN, Louis

1941 Catalogue de l'exposition précolombienne organisée à l'occasion du IIIe Congrès des Caraibes sous le haut patronage de Son Excellence M. Sténio Vincent. Port-au-Prince.

MANIGAT, Charles, MOÏsE, Claude \& OLLIVIER, Émile

1975 Haïti, quel développement? (Propos sur l'enquête... de Jean-Jacques Honorat). Montréal, Collectif Paroles.

MARS, Louis

1947 « L'hygiène mentale et la communauté haïtienne », Bulletin du Bureau d'ethnologie, II-3.

1970 « La psychiatrie au service du Tiers-Monde », Revue de la Faculté d’Ethnologie, 15.

MAXIMILIEN, Louis

1943 « Quelques apports indiens à la vie haïtienne », Les Cahiers d'Haïti, 5: 42-47. 
MINTZ, Sidney

1960 « Le système du marché rural dans l'économie haïtienne », Bulletin du Bureau d'ethnologie

d'Haïti, III-23-24-25.

NICHOLLS, David

1988 From Dessalines to Duvalier. Race, Colour and National Independence in Haiti. London, Macmillan Publishers (1re éd. 1979).

ORIOL, Jacques

1958 « In Memoriam Lorimer Denis », Bulletin du Bureau d'ethnologie, III-14.

1965 « Bientôt un quart de siècle », Bulletin du Bureau d'ethnologie, IV-31: 67-74.

1986 « Apport du Dr Louis Price-Mars à l'anthropologie sociale et culturelle haïtienne », Bulletin du Bureau national d'ethnologie: 15-30.

ORIOL, Jacques, VIAUD, Léonce \& AUBOURG, Michel

1952 « Le mouvement folklorique en Haïti », Bulletin du Bureau d'ethnologie, II-9.

PAUL, Emmanuel C.

1949 L'Ethnographie en Haïti. Ses initiateurs, son état actuel, ses tâches et son avenir. Port-au-Prince, Imprimerie de l'État.

1951 Nos chansons folkloriques et la possibilité de leur exploitation pédagogique. Port-au-Prince, Imp. Les Presses Libres.

1956 « Folkore et éducation », Bulletin du Bureau d'ethnologie, II-13.

1959 « Tâches et responsabilités de l'ethno-logie », Bulletin du Bureau d'ethnologie, III-17-18-19: 11-19.

PAUL, Max

1985 « En guise d'éditorial : culture et développement, une problématisation », Bulletin du Bureau national d'Ethnologie, 2: 5-6.

1990 « El Desarrollo de las investigaciones arqueológicas y etnológicas en Haïtí », in Pueblos y Políticas en el Caribe Amerindio. Memoria del Primer Encuentro del Caribe Amerindio. Santo Domingo, República Dominicana, 11-17 de septiembre de 1988. México, Instituto Indigenista Interamericano Fundación Garcia Arévalo: 43-49. (Trad. en espagnol d'Antoinette NelkenTerner.)

PIERRE, Claude, SATYRE, Joubert \& TROUILLOT, Lyonel

1993 « La Revue Indigène et la critique de l'indigénisme », Conjonction, 197: 55-63.

PRESSOIR, Charles Fernand

1947 Débat sur le créole et le folklore. Port-au-Prince, Imprimerie de l'État.

1958 «L'avenir du créole dans notre pays », Bulletin du Bureau d'ethnologie, III-16.

PRICE-MARS, Jean

1951 Folklore et patriotisme. Port-au-Prince, Imp. Les Presses Libres.

1954 Le Bilan des études ethnologiques en Haïti et Le Cycle du nègre. Port-au-Prince, Imprimerie de l'État. 
1998 Ainsi Parla l'Oncle. Port-au-Prince, L'Imprimeur II (1re éd. 1928).

ROMAIN, Jean-Baptiste

1984 « Recherche scientifique et technique dans le développement d'Haïti. Compilations et notes », Revue de la Faculté d'Ethnologie et du CHRESH, 42.

ROUMAIN, Jacques

1943 « Le musée du Bureau d'ethnologie », Rapport trimestriel du Bureau d'ethnologie, II-3: 34-35.

SOUFFRANT, claude

1986 « La société haïtienne, une société de la faim », Bulletin du Bureau d'ethnologie, 1-2: 35-51.

SUPPLICE, Daniel

2001 Dictionnaire biographique des person-nalités politiques de la République d'Haïti : 1804-2001.

Belgique, Imprimerie Lannoo.

TROUILLOT, Michel-Rolph

1993 « Jeux de mots, jeux de classe: les mouvances de l'indigénisme », Conjonction, 197: 29-45.

VIAUD, Léonce

1952 « Les activités du Bureau », Bulletin du Bureau d'ethnologie, II-9: 97-114

1958 « Les activités du Bureau », Bulletin du Bureau d'ethnologie, III-16: 54-57.

\section{ANNEXES}

\section{Témoignages et opinions sur les travaux du Bureau d'Ethnologie}

Roger Bastide, Lettre du 5 octobre 1948 au sujet de « L'organographie haïtienne » de Lorimer Denis et Emmanuel C. Paul

André Schaefner, Lettre à Lorimer Denis, 13 octobre 1948

Michel Leiris, Compte rendu du livre Mythologie vodou de Milo Marcellin, parue dans Le Mois d'ethnographie française, vol. 16, novembre 1951.

Jacques Butterlin (géologue français) à propos du travail de Michel Aubourg, dont il fut le professeur de préhistoire

Note du Bureau d'ethnologie à propos de Felix Coluccio, Folkloristas e Instituciones folkloricas del Mundo-Libreria. Buenos Aires, El Ateneo Editorial, 1951.

Aimé Césaire, Lettre à Lorimer Denis, 12 novembre 1944

Alejo Carpentier fait l'éloge de l'ethnologie en Haïti, du Bureau et de son musée ( $E l$ Nacional, Caracas, 11 sept. 1951). Traduction publiée dans le Bulletin, avril 1952, n 9, p. 113. 


\section{Liste des entretiens avec des personnalités haïtiennes:}

Blot, Jean Yves (10 avril 2003)

Dormilus, Arnold (18 octobre 2002)

Hector, Michel (9 décembre 2002)

Honorat, Michel Lamatinière (21 et 28 novembre 2002 ; 14 avril 2003)

Mangonès, Lorraine (6 décembre 2002)

Oriol, Michèle (2 avril 2003)

Paul, Max (11 et 21 avril 2003)

Paul, Waltraud Grohs (12 novembre $2002 ; 11$ avril et 21 avril 2003)

Smarth, Luc (8 avril 2003)

Smarth, Lucien (9 janvier 2003)

Vilaire, Patrick (17 avril 2003).

\section{NOTES}

1. Je me réfère, sous ce nom, à une même institution connue sous deux appellations: Bureau d'ethnologie de la République d'Haïti, de 1941 à 1984, puis Bureau national d'ethnologie de 1984 à aujourd'hui. Par commodité, j'utilise ici le terme Bureau d'ethnologie ou plus simplement le Bureau.

2. Pour une filiation idéologique plus complète du mouvement ethnographique et folklorique haïtien, il faudrait remonter au $\mathrm{XIX}^{\mathrm{e}}$ siècle. Pour des informations détaillées, cf. Price-Mars (1954) ; Oriol, Viaud \& Aubourg (1952) ; Nicholls (1988).

3. En Haïti, on appelle génération de la "gifle» les personnes ayant vécu sous l'occupation américaine et qu'on retrouve dans les mouvements de mobilisation sociale, politique et littéraire contre l'occupant.

4. Les autres membres de la Revue Indigène sont: Carl Brouard, Arthur Bonhomme, Kléber Georges Jacob.

5. Aujourd'hui la réputation de la Revue Indigène paraît surfaite: certains critiques (en particulier Claude Pierre, Joubert Satyre et Lyonel Trouillot, 1993) contestent que ses membres aient eu des préoccupations sociales et politiques. Pour une nouvelle approche de la question, voir les deux numéros de la revue Conjonction (1993, nº 197 et $\mathrm{n}^{\circ}$ 198) consacrés à l'indigénisme haïtien.

6. Roger Gaillard (1993 : 9) définit ainsi l'indigénisme haïtien: «En Haïti, on appelle indigénisme la volonté, chez les créateurs esthétiques, de s'inspirer des coutumes, des valeurs appartenant à la vie, à la culture nationale. »

7. Voir l'analyse éclairante de D. Nicholls (1988).

8. Jean Price-Mars (1954: 20) écrit: « ...créé par l'État sous les instances, les démarches et les inspirations de Jacques Roumain ».

9. Entretien avec Michel L. Honorat, Pétionville, 21 novembre 2002. 
10. L'explication peut être jugée insuffisante, mais elle fournit des éléments de réponse. Entre 1926 et 1936, Roumain avait été arrêté plusieurs fois pour activités subversives diverses; en 1932, il avait fondé le premier Parti communiste haïtien ( $\mathrm{PCH}$ ).

11. La conception du Bureau d'ethnologie haïtien n'est pas sans rappeler celle du Bureau of American Ethnology, qui a des liens organiques avec la Smithsonian Institution de Washington. Ces deux institutions se sont impliquées en Haïti dès la fin du XIX ${ }^{\mathrm{e}}$ siècle.

12. D'après le rapport annuel 1942-43 de Rémy Bastien, il est certain que les premières collections ethnographiques et archéologiques du Bureau ont été alimentées en grande partie par cette fameuse " campagne anti-superstitieuse ».

13. Cf. David Nicholls (1988). De surcroît, les contemporains des deux hommes affirment que l'estime était réciproque: J. Price-Mars appelait Roumain son « fils spirituel » et avait préfacé son roman La Montagne ensorcelée.

14. Nous sommes alors en 1959, à la veille des mouvements de décolonisation en Afrique et dans la Caraïbe, et des luttes pour la conquête des droits civiques et politiques des Noirs aux ÉtatsUnis.

15. C'est nous qui soulignons.

16. J. Oriol (1965: 68) assume la continuité de la mission: « Combattre sur le plan éducatif jusqu’à l'extirpation complète les tares héritées de l'ancienne société esclavagiste de Saint-Domingue. En un mot, une véritable entreprise de désaliénation de notre collectivité, telle que l'a forgée l'histoire, pour un meilleur devenir. "

17. Pour la période 1941-1963, voir la compilation des sommaires des différents Bulletins et Publications effectuée par Charles Arnoux (1964) et, pour la période suivante, le répertoire bibliographique établi par Arnold Dormilus (1995-1997).

18. Il est intéressant de noter la distinction établie à l'origine entre bulletins et publications du Bureau. Cette distinction semble se baser surtout sur le public visé et sur le mode de diffusion. Les Bulletins devaient être distribués gratuitement, probablement comme outils de sensibilisation et de vulgarisation, tandis que les Publications, destinées à un public de spécialistes et financées par leurs auteurs, devaient être vendues. Quant à la numérotation des différentes séries, elle n'est pas toujours systématique, ce qui peut prêter à confusion.

19. Sur l'influence de la Seconde Guerre mondiale sur le Bureau, voir Bastien (1944).

20. M. Aubourg (1951). Pour une présentation plus succincte du développement des études archéologiques en Haïti, voir aussi Max Paul (1990).

21. Louis Maximilien (1943) fait référence au Manuel d'histoire d'Haïti de Justin Chrysostome Dorsainvil qui venait d'être publié. Rédigé en collaboration avec les frères de l'Instruction chrétienne (clergé français), ce manuel occupe encore aujourd'hui une place prépondérante parmi les manuels scolaires haïtiens, contribuant à alimenter la vision que des générations d'écoliers se font de leur histoire.

22. En 1958, trois articles paraissent dans le Bulletin, dont les auteurs sont Odnell David, Michelson P. Hyppolite et Charles Fernand Pressoir. À noter que l'Institut d'ethnologie fut le premier à introduire l'enseignement de la linguistique en Haïti.

23. Des recherches avaient été menées, dès 1944, sur le marché de Beaumont par une étudiante de Yale, et les résultats furent publiés aux États-Unis. Le Bureau ne semble pas en avoir eu connaissance. En fait, dès la fin des années 1930 et pendant les années 1940, les chercheurs étrangers s'intéressent à Haïti, mais leurs publications n'ont pas toutes été envoyées au Bureau.

24. Ce fut le cas de Michel L. Honorat, Rémy Bastien, Roger Mortel qui, frais émoulus de l'Institut d'ethnologie de Port-au-Prince, participèrent à l'enquête ethnographique menée par l'Unesco à Marbial (sud-est du pays). Ils étaient encadrés en particulier par Jean Comhaire et son épouse, Suzanne Comhaire-Sylvain, ainsi que par Alfred Métraux (M. L. Honorat, communication personnelle, 28 Novembre 2002). 
25. Jacques Roumain (mort à 37 ans, deux ans à la tête du Bureau), Lorimer Denis (mort à 53 ans, onze ans de gestion), Emmanuel C. Paul (mort à 50 ans, six ans de gestion).

26. J'emprunte l'expression à Michèle Oriol (communication personnelle, 2 avril 2003).

27. Cf. Oriol (1986) et Mars (1970: 40).

28. Voir les critiques de Manigat, Moïse \& Ollivier (1975); voir aussi Souffrant (1986 : 38-39).

29. Par exemple, réaction du journal Le Soir rapportée par G. Corvington (1991: 324) : « Le Soir réprouvait l'école intellectuelle qui se donne pour tâche de réhabiliter aux yeux de la civilisation nos pires traditions ancestrales, croyant les ennoblir en en faisant l'objet de considérations scientifiques et en leur donnant une place dans la terminologie occulte. »

30. Cf. le communiqué du sous-secrétaire d'État de l'information établissant une censure des scènes de rituel vodou dans les pièces de théâtre (Corvington 1991: 195).

31. L'un des directeurs interrogés témoigne que le Bureau a été l'objet de menaces après 1986. En outre, un incendie suspect, aux causes non déterminées, a détruit les archives de la Faculté d'ethnologie.

32. L'utilisation politique du vodou par François Duvalier est bien connue et documentée (Laguerre 1982). Nombre de oungan (prêtres vodou) étaient aussi chefs de Tontons macoutes (milice de Duvalier). Après la chute de Duvalier, la vindicte populaire s'en est prise à eux et, lors des dechoukaj de 1986 (actes de représailles populaires où les présumés partisans d'un régime déchu sont exécutés sommairement), nombres d'entre eux ont été assassinés.

33. Un témoin de l'époque, proche des deux hommes, souligne que L. Denis, par esprit de fidélité, ajouta le nom de Duvalier à certains de ses travaux, bien que celui-ci n'y ait pas collaboré, se trouvant à l'étranger en voyage d'études (M. L. Honorat, communication personnelle, 14 avril 2003). La plupart des articles co-signés ont paru dans Les Griots. En fait, Duvalier ne publia que deux articles co-signés dans le Bulletin du Bureau (en 1947 et en 1955) et Denis signa seul tous ses autres articles publiés dans le Bulletin.

34. Dans le Dictionnaire des personnalités politiques d'Haïti, de Daniel Supplice, on retrouve les ethnologues suivants, avant Duvalier : J. Price-Mars, J. Roumain, E. Mangonès, E. C. Paul ; période de Duvalier : E. C. Paul, J. Oriol, M.L. Honorat, L. Viaud.

35. Par exemple, le puissant commandant des Casernes Dessalines, Jean-Claude Paul.

36. Il est important de rappeler que Lorimer Denis est mort en août 1957, un mois avant l'élection de François Duvalier à la présidence d'Haïti, alors que la rumeur publique fait de lui un «duvaliériste-macoute ».

37. Un léger toilettage fut effectué en 1992 par les pouvoirs publics pour qu'il devienne pendant trois jours "la maison du Roi du Carnaval», ce qui, pour le directeur d'alors, Lucien Smath, représentait un détournement choquant de la vocation scientifique et intellectuelle du Bureau.

\section{RÉSUMÉS}

L'article pose la question de l'engagement en sciences sociales et du rapport entre anthropologie, politique et société en Haïti. La définition de la mission du Bureau d'ethnologie d'Haïti, créé en 1941 pour étudier et défendre la culture populaire, donna lieu à des débats qui sont loin d'être épuisés aujourd'hui. Le Bureau proposait une nouvelle définition de la «culture haïtienne ", où prédominait l'apport africain, contrairement à la définition des classes dominantes pour qui l'apport européen constituait l'élément essentiel. Après avoir retracé l'histoire intellectuelle et 
institutionnelle du Bureau d'ethnologie, ses moments de gloire et de misère, l'article suggère que la vision essentialiste à tonalité fortement raciale de la culture haïtienne proposée par les ethnologues de l'époque donna lieu à une récupération idéologique par le gouvernement de François Duvalier, ce qui contribua pour beaucoup à la persistance de l'image négative du Bureau d'ethnologie qui est encore la sienne aujourd'hui.

The Bureau d'Ethnologie in Haiti. - What are the relations between anthropology, politics and society in Haiti? How have the social sciences been involved? Haiti's Bureau d'Ethnologie was set up in 1941 to study and defend folk culture; this assignment has aroused debates that are still far from settled. The Bureau proposed a new definition of Haitian culture with preeminence given to African aspects, unlike in the definition made by the ruling classes for whom European traits were the main ingredient. After presenting the Bureau's intellectual and institutional history with its hours of glory and of woe, the suggestion is made that, as proposed by ethnologists at the time, this essentialistic vision of Haitian culture with strong racial overtones laid the grounds for an ideological recuperation by François Duvalier's government. This accounts for the Bureau d'Ethnologie's still persisting negative image.

INDEX

Mots-clés : Haïti, histoire de l'ethnologie, identité haïtienne, institutions culturelles, institutions postcoloniales

Keywords : cultural institutions, Haitian identity, history of ethnology, postcolonial institutions

\section{AUTEUR}

\section{RACHELLE CHARLIER-DOUCET}

Université Wilfrid Laurier Département d'anthropologie Waterloo, ON, rdoucet@wlu.ca 